\title{
¿18 años no es nada? Una nueva revisión del uso correcto, parcial e incorrecto de los términos 'hipótesis' y 'predicciones' en ecología
}

\author{
Alejandro G. Farji-Brener \\ Laboratorio de Investigaciones en Hormigas (LIHO), INIBIOMA, CONICET y Universidad Nacional del Comahue. Bariloche, \\ Argentina.
}

\begin{abstract}
Resumen. En 2003 publiqué un artículo en Ecología Austral en el que revelaba que la gran mayoría de sus manuscritos empleaban de forma incorrecta los términos hipótesis y predicciones. En dicho artículo explicaba y ejemplificaba los motivos de dicha confusión, describía los conceptos básicos del método hipotético-deductivo (MHD) y finalizaba con algunos consejos para su buen uso. En este trabajo evalué nuevamente el empleo adecuado del MHD en los manuscritos publicados en Ecología Austral a posteriori de la aparición de aquel artículo, para determinar la influencia potencial de la revisión original a 18 años de ser publicada. Examiné un total de 691 manuscritos publicados entre 2015 y 2019; 241 de Ecología Austral, 152 de Oikos y 298 de Ecology. Comparados con los resultados del período analizado en el trabajo original (1997-2002), las publicaciones en Ecología Austral que emplearon el MHD se duplicaron, mientras que aquellas que empleaban correctamente los términos hipótesis y predicciones aumentaron 7 veces. Tal incremento no respondió a una tendencia general en las publicaciones ecológicas, ya que no lo detecté en las revistas anglo-parlantes que analicé. Este aumento en las publicaciones de Ecología Austral que emplearon el MHD entre 2015-2019 y utilizaron de forma adecuada su terminología asociada sugiere una influencia positiva de la revisión original del 2003. En el presente trabajo discuto hipótesis alternativas que también podrían reflejar este patrón. Dado el número relativamente bajo de citas de Farji-Brener (2003), estos resultados cuestionan la precisión del número de citas como un estimador del impacto de ciertos manuscritos e ilustran la importancia de escribir artículos en español que colaboren con el desarrollo de las investigaciones en ecología, aunque normalmente sean subvalorados en las instancias formales de evaluación académicas.
\end{abstract}

[Palabras clave: investigación científica, método hipotético-deductivo]

Aвstract. Are 18 years really nothing? An updated review of the correct, partial and incorrect use of the terms 'hypotheses' and 'predictions' in ecology. In 2003, I published an article in Ecología Austral revealing that most manuscripts used the terms hypotheses and predictions incorrectly. That article explained and exemplified the reasons for that confusion, described the basic concepts of the hypothetical-deductive method (MHD) and ended with some guidelines for its proper use. Here, I evaluated again the adequate use of MHD in the manuscripts published in Ecología Austral after the publication of that paper, to determine the potential influence of the original revision 18 years after its publication. I reviewed a total of 691 articles published between 2015 and 2019; 241 in Ecología Austral, 152 in Oikos and 298 in Ecology. Compared with the results of the period analyzed in the original work (1997-2001), the publications in Ecología Austral that used the MHD doubled, while those that correctly used the terms hypotheses and predictions showed a 7-fold increase. This increase did not respond to a general trend in ecological publications, since it was not detected in the English-speaking journals analyzed. These results suggest a positive influence of the original revision where these concepts were discussed in 2003. Alternative hypotheses are discussed that could also cause this pattern. Given the relatively low number of citations of Farji-Brener (2003), these results question the citation number as a precise estimator of the impact of certain kind of manuscripts, and illustrate the importance of writing articles in Spanish that help the development of research in ecology, although normally are undervalued in formal instances of academic evaluation.

[Keywords: scientific research, hypothetical-deductive method]

Editor asociado: Ricardo Grau 


\section{INTRODUCCIÓN}

Una de las maneras más potentes que tenemos los ecólogos para conocer las causas de los patrones naturales es poner a prueba ideas sobre los posibles mecanismos que los generan (Lawton 1996). Dado que las ideas sólo pueden probarse a través de sus consecuencias, para determinar el origen de un patrón debemos proponer sus posibles causas (i.e., hipótesis), deducir sus consecuencias suponiendo que dichas causas son verdaderas (i.e., proponer predicciones) y contrastar los resultados propuestos por esas predicciones con aquellos observados en nuestra investigación. Esta metodología, denominada método hipotético-deductivo (MHD) porque se proponen hipótesis y se deducen sus consecuencias, nos permite descartar las hipótesis menos probables y quedarnos con aquella a la que transitoriamente se la considera verdadera. A través de esta forma de descartar hipótesis teóricamente erróneas se avanza en la construcción del conocimiento (Bunge 2002). Pese a la relativa sencillez de su razonamiento, el empleo del MHD en el mundo hispano-parlante generó una gran cantidad de publicaciones sobre sus virtudes y limitaciones potenciales (Marone and González del Solar 2000; Bunge 2002; Marone and Galetto 2011; Feinsinger 2013, 2014; Farji-Brener and Amador-Vargas 2014; FarjiBrener 2019). Independientemente de dichas controversias, al MHD se lo suele aceptar como una herramienta útil si se quieren poner a prueba ideas explicativas (Marone and Galetto 2011; Farji-Brener 2019).

Durante muchos años, al dictar cursos de ecología de campo fui testigo de lo difícil que era para los estudiantes planificar proyectos de investigación empleando el MHD. Algunas veces intentaban transformar un buen estudio descriptivo en uno deductivo (con pésimas consecuencias), formulaban hipótesis de manera errónea o incompleta, y enunciaban predicciones como si fuesen hipótesis. Dicha experiencia me llevó a escribir un artículo en el que explicaba las diferencias conceptuales entre los términos hipótesis y predicciones, y revisaba su uso correcto, parcial e incorrecto en las publicaciones de Ecología Austral y de otras revistas anglo-parlantes similares (FarjiBrener 2003). Los resultados de dicho análisis revelaron que la gran mayoría de los artículos publicados en Ecología Austral durante el período 1997-2002 no empleaban el MHD, y que los pocos que lo empleaban, lo hacían de forma parcial o incorrecta. El artículo finalizaba con una serie de comentarios, ejemplos y sugerencias para que se comprendieran mejor las diferencias conceptuales entre los términos hipótesis y predicciones, y que se emplearan de forma correcta. A pesar de su relativamente escaso número de citas ( 24 al día de la escritura de este manuscrito según Google Scholar), en todos estos años recibí numerosos comentarios de estudiantes indicando que lo habían tenido como literatura de cabecera al planificar sus trabajos de investigación, y sé que fue material de lectura en muchos cursos de grado y posgrado en universidades nacionales y extranjeras de habla hispana. En consecuencia, y luego de 18 años de haberse publicado dicho artículo, considero que es momento de volver a revisar el uso de los términos hipótesis y predicciones en los manuscritos publicados en Ecología Austral, para determinar si el artículo original (Farji-Brener 2003) tuvo un impacto en la adopción y el uso correcto del MHD desde su publicación o si, como en el tango, "todo sigue igual que ayer".

\section{Materiales y Métodos}

Para determinar el uso correcto, parcial o incorrecto de los términos hipótesis y predicciones revisé todos los manuscritos publicados en Ecología Austral desde el 2005 hasta el 2019 (41 volúmenes), y en los 12 volúmenes del año 2015 de Ecology y Oikos. Estas revistas anglo-parlantes se emplearon, como en el artículo del 2003, como una especie de 'control' para determinar si los patrones encontrados en Ecología Austral respondían a una tendencia general en las publicaciones académicas en la disciplina. Utilicé los volúmenes del año 2015 de Oikos y Ecology porque a partir de ese año detecté un pico de publicaciones en Ecología Austral que empleaban el MHD de forma correcta (ver resultados). Al igual que en el artículo original, los manuscritos de revisión, números especiales, de conceptos o síntesis, y las notas cortas o comentarios no fueron tenidos en cuenta para este estudio. En total analicé 691 manuscritos (241 de Ecología Austral, 152 de Oikos y 298 de Ecology). Para cada manuscrito primero determiné si los autores empleaban el MHD (i.e., si pretendían poner a prueba alguna hipótesis) o si era un estudio descriptivo (i.e., pretendían responder preguntas específicas o determinar un patrón). El uso del MHD fue detectado a través de la lectura completa de la introducción de los manuscritos, independientemente si las hipótesis y predicciones eran presentadas explícitamente 
(e.g., "nuestra hipótesis de trabajo es...") o su existencia se desprendía de manera implícita a partir de la lectura del texto. Luego, en el sub-conjunto de todos los manuscritos que empleaban el MHD determiné si los términos hipótesis y predicciones eran empleados correcta, parcial o incorrectamente según los mismos criterios usados en Farji-Brener (2003). Se consideró uso incorrecto cuando las hipótesis se planteaban como predicciones (i.e., como resultados esperados y no como ideas $\mathrm{u}$ opiniones), cuando las predicciones eran enunciadas sin explicitar las hipótesis que las generaban, o cuando las predicciones planteadas no eran consecuencias lógicas inequívocas de las hipótesis. Estos sesgos impiden conocer la causa o mecanismo que realmente se está poniendo a prueba, y limitan juzgar críticamente la coherencia lógico-deductiva del estudio. Uso parcial se consideró cuando se planteaban hipótesis sin predicciones, sesgo que impide conocer los resultados esperados que el autor pondrá a prueba para validar su idea. Finalmente, se calificó con uso correcto aquellos manuscritos que claramente planteaban hipótesis y sus predicciones asociadas de forma explícita o implícita (ver detalles en Farji-Brener 2003). Todas las comparaciones se realizaron empleando pruebas de independencia $\left(X^{2}\right)$ utilizando la corrección de Yates cuando era necesario (i.e., tablas de $2 \times 2$ ), y empleando los $\mathrm{X}^{2}$ parciales para determinar la importancia relativa de los efectos sobre el $\mathrm{X}^{2}$ total (Monroy et al. 2018).

\section{Resultados}

La proporción de manuscritos que emplearon el MHD durante el período 2005-2019 fue levemente menor en Ecología Austral que los publicados en Oikos y Ecology durante 2015. De los 241 manuscritos publicados en Ecología Austral, 94 (39\%) emplearon el MHD, mientras que de los 152 y 298 aparecidos en Oikos y en Ecology, usaron el MHD 64 (42\%) y $148(50 \%)$ publicaciones, respectivamente $\left(X^{2}=6.5\right.$, g.l. $\left.=2, P=0.04\right)$. Sin embargo, Ecología Austral fue la única de las revistas analizadas que incrementó en el tiempo la proporción de manuscritos que emplearon el MHD (Figura 1). Por un lado, mientras que en Ecología Austral el uso del MHD subió del 21\% al 39\% desde el período 1997-2002 al 2005-2019 (14/67 versus $94 / 241$ trabajos, respectivamente; $X^{2}=6.8$, g.l. $=1, P=0.009$ ), tanto en Oikos como en Ecology la proporción de trabajos que emplearon el MHD se mantuvo similar
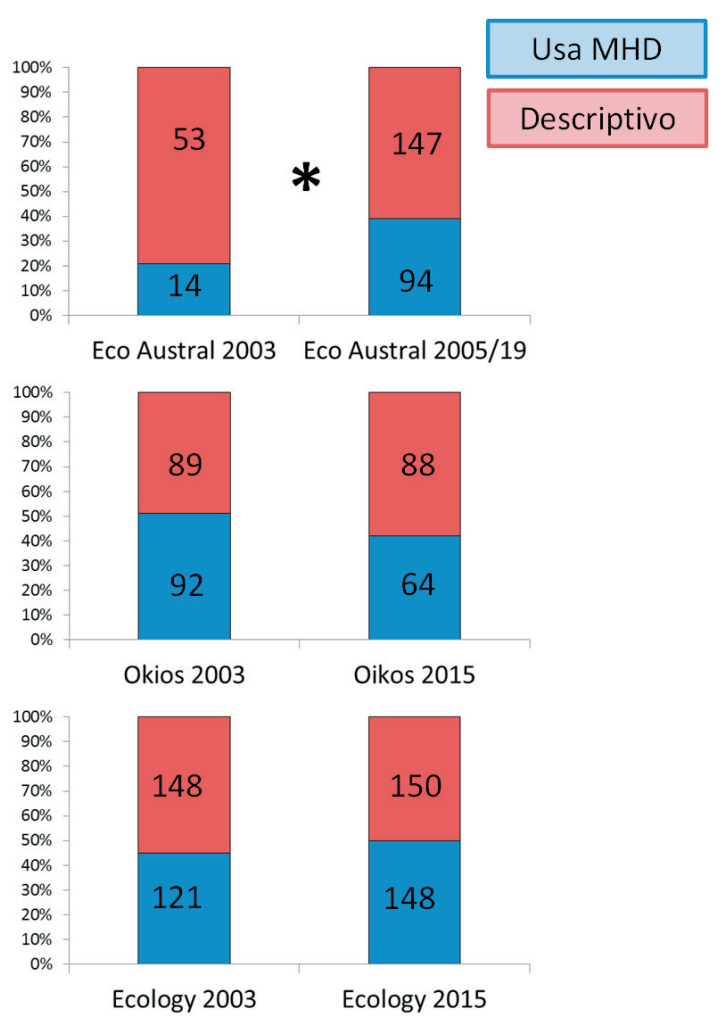

Figura 1. Porcentaje de trabajos que usan el método hipotético deductivo (MHD) o son de naturaleza descriptiva (i.e., responden preguntas o describen patrones) en las revistas Ecología Austral, Oikos y Ecology analizados en Farii-Brener (2003) y en la revisión actual. Los números dentro de las barras son la cantidad absoluta de trabajos analizados en cada categoría. El símbolo * representa diferencias estadísticamente significativas $(P<0.05)$.

Figure 1. Percentage of articles that use the hypothetical deductive method (MHD) or are descriptive (i.e., answer questions or describe patterns) in the journals Ecología Austral, Oikos and Ecology analyzed in Farji-Brener (2003) and in the current review. The numbers inside the bars are the absolute number of manuscripts analyzed in each category. The symbol ${ }^{*}$ represents statistically significant differences $(P<0.05)$

entre los períodos analizados (de $51 \%$ al $42 \%$ y del $45 \%$ al $50 \%, X^{2}=2.2$ y 1.1., g.l. $=1$, $P=0.14$ y 0.30 , respectivamente) (Figura 1 ). Este aumento en la proporción de trabajos publicados en Ecología Austral que usaron el MHD fue creciendo en el tiempo desde el 2005 al 2019, con un pico a partir del 2015 (Figura 2). Considerando sólo los manuscritos que empleaban el MHD, alrededor del 50\% de dichos artículos emplearon en forma incorrecta o parcial los términos hipótesis y predicciones en todas las revistas analizadas (Figura 3). El uso incorrecto más frecuente fue presentar predicciones como si fuesen hipótesis (ver algunos ejemplos en la Tabla $1)$, seguido por presentar predicciones sin 


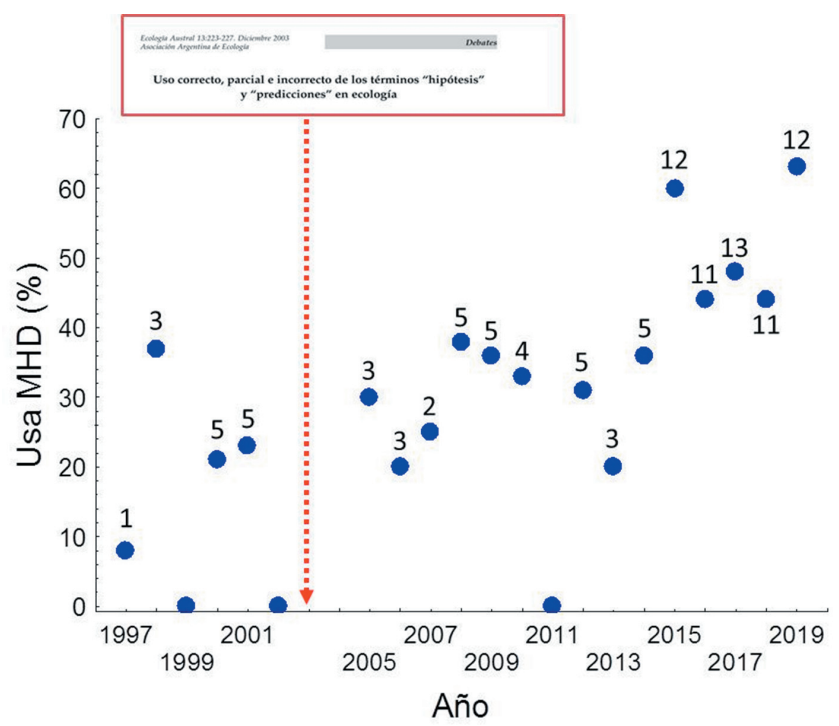

Figura 2. Porcentaje de trabajos que emplean el método hipotético deductivo (MHD) publicados en Ecología Austral entre 1997 y 2019. La flecha roja indica el año de la publicación del artículo original que discutía el adecuado uso del MHD (Farji-Brener 2003). Los números arriba de cada punto indican el número absoluto de manuscritos.

Figure 2. Percentage of articles using the hypothetical deductive method (MHD) published in Ecología Austral between 1997 and 2019. The red arrow indicates the year of publication of the original article that discussed the proper use of MHD (Farji-Brener 2003). The numbers above each point indicate the absolute number of manuscripts.
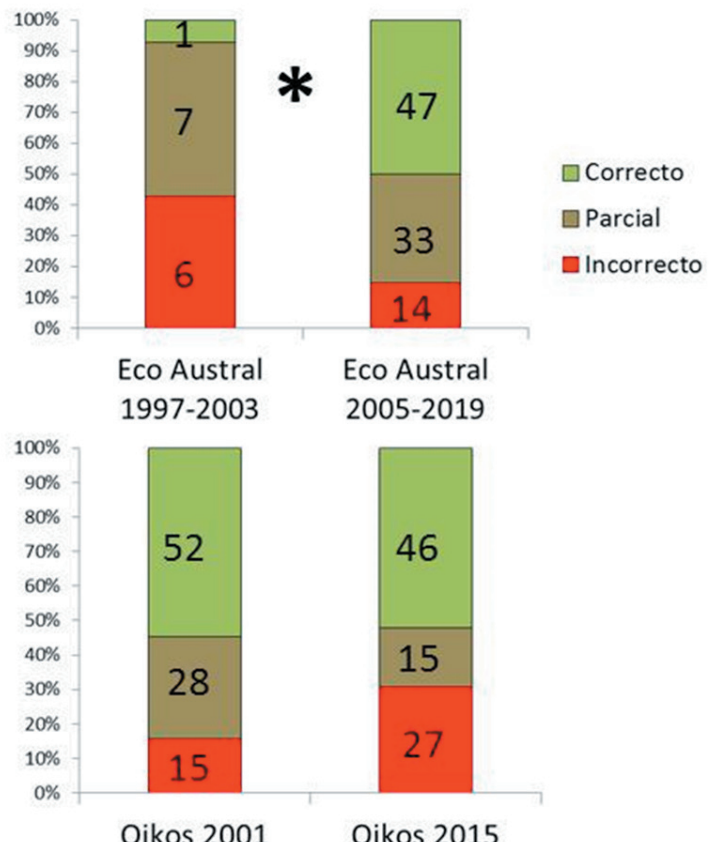

Figura 3. Porcentaje de trabajos que usan los términos hipótesis y predicciones de forma correcta, parcial o incorrecta en las revistas Ecología Austral, Oikos y Ecology analizados en Farji-Brener (2003) y en la revisión actual. Los números dentro de las barras son la cantidad absoluta de trabajos analizados en cada categoría. Sólo en Ecología Austral hay un incremento estadísticamente significativo ${ }^{*}$ ) en el porcentaje de artículos que usan correctamente los términos.

Figure 3. Percentage of articles that use the terms hypotheses and predictions correctly, partially or incorrectly in the journals Ecología Austral, Oikos and Ecology analyzed in Farji-Brener (2003) and in the current review. The numbers inside the bars are the absolute number of manuscripts analyzed in each category. Only in Ecología Austral we found a statistically significant increase $\left(^{*}\right)$ in the percentage of articles that correctly use the terms. 


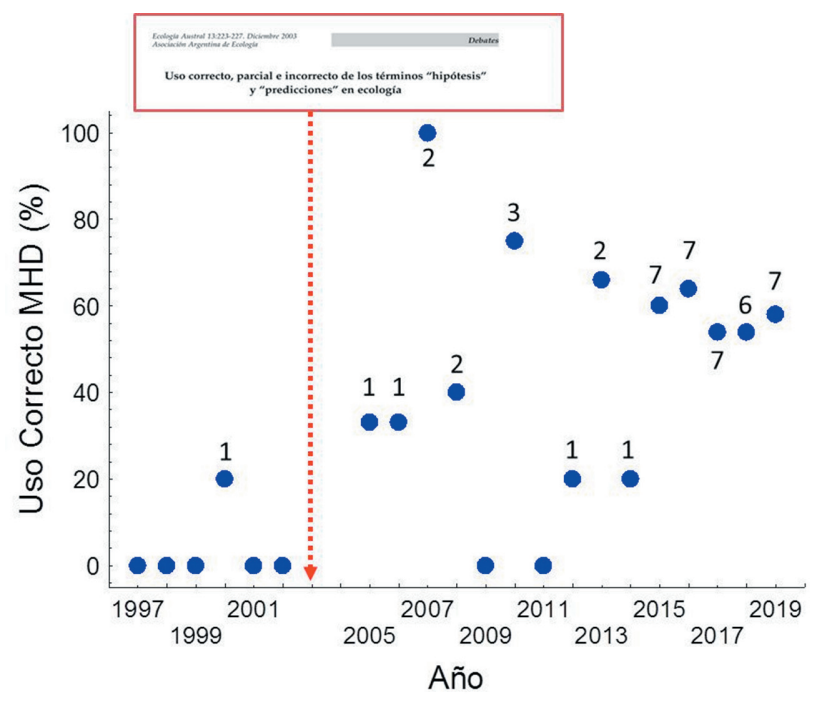

Figura 4. Porcentaje de manuscritos que emplean correctamente los términos hipótesis y predicciones en Ecología Austral entre 1997 y 2019. La flecha roja indica el año de la publicación del artículo original que discutía el adecuado uso del MHD (Farji-Brener 2003). Los números arriba de cada punto indican el número absoluto de manuscritos.

Figure 4. Percentage of articles that correctly use the terms hypotheses and predictions published in Ecología Austral between 1997 and 2019. The red arrow indicates the year of publication of the original article that discussed the proper use of MHD (Farji-Brener 2003). The numbers above each point indicate the absolute number of manuscripts.

Tabla1. Algunos ejemplos textuales de usos considerados incorrectos de los términos hipótesis y predicciones en las revistas Ecología Austral, Oikos y Ecology. En todos estos casos, el problema es que los autores proponen resultados esperados (i.e., predicciones) como si fuesen hipótesis, por lo cual se desconoce la idea o concepto que se está poniendo a prueba.

Table 1. Some textual examples of incorrect uses of the terms hypotheses and predictions in the journals Ecología Austral, Oikos and Ecology. In all cases, the problem is that the authors propose expected results (i.e., predictions) as if they were hypotheses, so the conceptual idea being tested is unknown.

Ecología Austral

- Como hipótesis se plantea que los micrositios con acumulación de materia orgánica tienen bancos de semillas con mayor tamaño y composición que los micrositios sin acumulación

- Las hipótesis propuestas son: a) los talares albergan una comunidad de oribátidos más diversa y abundante que en la matriz circundante

- La hipótesis de trabajo del presente estudio establece que la eficiencia de reabsorción de nitrógeno es mayor en Nassella tenuissima que en Poa ligularis

- Se plantearon las siguientes hipótesis de trabajo: i) los individuos de M. tridens rebrotan luego del corte de la parte aérea...

Oikos

- We followed three hypotheses: 1) seedlings grown both at temperatures characteristic of lower elevations and in soils from lower elevations will grow larger than seedlings grown at temperatures and in soils from higher elevations...

- We specifically sought to test the following hypotheses: 1) tree height growth is higher in mixed tree communities compared to monocultures...

Ecology

- We hypothesized that (1) increasing plant diversity leads to increasing soil microbial biomass and enzyme activity,

(2) higher temperature decreases soil microbial biomass and enzyme activity

- We hypothesized that steep slopes would have substantially lower N-availability than the relatively flat, stable ridge tops

- We hypothesized that nutrient addition would lead to reductions in fine-root biomass, diameter, tissue density, and the abundance of AM fungal structures 
mencionar las hipótesis que las generaban. Sin embargo, Ecología Austral fue la única revista que mostró un incremento en la proporción de manuscritos publicados que emplearon correctamente los términos hipótesis y predicciones, alcanzando los niveles de las revistas anglo-parlantes analizadas. Mientras que en Ecología Austral dichos manuscritos aumentaron del 7\% al 50\% desde 1997-2002 al 2005-2019 (de 1/14 a 47/94, $X^{2}=11, P=0.009$ ) (Figuras 3 y 4), tanto en Oikos como en Ecology no mostraron grandes cambios y se mantuvieron alrededor del 50\% (57\% a 53\% y $46 \%$ a $48 \%$, respectivamente, $X^{2}=0.05$ y 0.002 , $P>0.60$ ) (Figura 3).

\section{Discusión}

Este trabajo revela un resultado sugerente: los manuscritos publicados en Ecología Austral que usan el MHD y emplean de forma correcta los términos hipótesis y predicciones se incrementaron en el período 2005-2019 comparado con el período 1997-2002. Elempleo del MHD creció casi el doble (21 al 39\%), mientras que el uso correcto de los términos hipótesis y predicciones aumentó 7 veces (del 7 al 50\%), ambos patrones particularmente notables desde 2015. Aparentemente, este incremento no responde a una tendencia general en las publicaciones ecológicas, ya que no fue detectado en las revistas angloparlantes analizadas. Tomado en conjunto, estos resultados sugieren que el artículo original donde se discutía la problemática del buen uso del MHD (Farji-Brener 2003) tuvo una influencia positiva sobre sus lectores, ya que a partir de su aparición se incrementaron los manuscritos publicados en Ecología Austral que emplearon adecuadamente el MHD. Este hipotético impacto mostró un tiempo de retardo de varios años, lapso consistente con el propuesto por algunos autores para evaluar el impacto de los manuscritos (Jaksic 1994; Olby 2003).

Pese a que detecté un claro incremento de los manuscritos que emplean de forma correcta el MHD en Ecología Austral desde la publicación de Farji-Brener (2003), la influencia de este trabajo sobre dicho patrón puede ser debatible. Primero, esta tendencia puede responder a otros motivos no analizados en este trabajo. Varias razones ajenas a la publicación de Farji-Brener (2003) pueden haber influido para que los manuscritos publicados en el período 2005-2019 hayan empleado de manera más adecuada el MHD. Por ejemplo, mejoras en la educación superior (e.g., la inclusión del empleo del MHD en materias ecológicas o la lectura de otros artículos sobre la temática) pueden haber favorecido el uso adecuado del MHD. Además, se desconoce si los autores que publicaron en Ecología Austral durante 20052019 necesariamenteleyeron el artículo de FarjiBrener (2003). Un cuestionario dirigido a los autores de los trabajos publicados durante este período en Ecología Austral podría confirmar esta hipótesis. En las últimas décadas pudo haber un aumento de publicaciones sobre el MHD que, independientemente del artículo de Farji-Brener (2003), hayan estimulado a desarrollar proyectos de investigación empleando de manera adecuada dicho método. Un rápido análisis en Google Scholar apoya parcialmente esta hipótesis alternativa. Empleando las palabras "método hipotético deductivo-ecología" encontré que la tasa de publicación sobre dicha temática fue mayor en el período 2005-2019 (324 trabajos/año) que durante el período 1997-2002 (46 trabajos/año). Este aumento en las publicaciones en idioma español sobre el MHD pudo haber influido de forma positiva en el incremento detectado en las publicaciones de Ecología Austral. Finalmente, el efecto 'positivo' de dicho artículo puede no haber sido sólo sobre los lectores, sino también sobre los editores y revisores de la revista, estimulando un control más riguroso e incentivando un uso adecuado del MHD para la publicación de manuscritos en Ecología Austral. Segundo, en este trabajo solo se evalúa el uso del MHD en Ecología Austral. Aunque es lógico analizar el impacto de un artículo en la misma revista adonde fue publicado, sería aconsejable incluir otras revistas sobre ecología de habla hispana para ampliar el estudio de sus potenciales efectos. Tercero, se evalúan solo dos revistas anglo-parlantes para detectar si el patrón encontrado en Ecología Austral es producto de una tendencia general en las publicaciones ecológicas. Incluir más publicaciones angloparlantes fortalecería el patrón encontrado en este estudio. Sin embargo, se emplearon las mismas revistas y se examinaron un número de manuscritos similar al realizado en el artículo original (535 versus 450$)$, sin encontrar tendencia alguna. En resumen, proponer que el incremento actual de manuscritos que usan adecuadamente el MHD en Ecología Austral sea una consecuencia del artículo que discutió esta temática por primera vez en 2003 puede ser demasiado simplista. Pero, dados los resultados encontrados en este trabajo, descartar o minimizar su impacto 
positivo sobre esta tendencia tampoco parece razonable.

El empleo correcto de los términos hipótesis y predicciones no es una simple cuestión semántica. Como se resumió antes y como se ha discutido detalladamente en otros artículos, postular predicciones como si fuesen hipótesis o plantear predicciones sin mencionar las ideas que teóricamente las generan son limitaciones conceptuales que impactan negativamente en el desarrollo, la puesta en práctica y la interpretación de las investigaciones en ecología y en ramas afines (Farji-Brener 2003; Farji-Brener and AmadorVargas 2014, 2018; Farji-Brener 2019). Sin embargo, el empleo del MHD no es la única herramienta para generar conocimiento. La ciencia también avanza con buenos trabajos descriptivos en los que se intenta responder preguntas específicas sin hipótesis previas, o se pretende describir o confirmar patrones (Lawton 1996; Farji-Brener 2019). No es la intención de este trabajo comparar el MHD con otras aproximaciones, ya que todos los métodos presentan fortalezas y debilidades (Farji-Brener 2019). Aquí sólo evalué el uso correcto de este enfoque epistemológico. Pero dado que el uso del MHD es reconocido como un enfoque poderoso para poner a prueba ideas generales sobre cómo funciona la naturaleza (Bunge 2002; Marone and Galetto 2011; Farji-Brener 2019), su aplicación correcta amerita una evaluación rigurosa. Más allá de las hipótesis alternativas planteadas en el párrafo anterior, si suponemos que el incremento en el uso adecuado del MHD en manuscritos aparecidos en Ecología Austral desde 2005 hasta la fecha es un reflejo parcial de la influencia del artículo publicado en 2003, sobresalen dos aspectos dignos de reflexión. Primero, estos resultados cuestionan el valor del número de citas de un artículo como una herramienta excluyente para evaluar su impacto, en particular en el caso de artículos de opinión y especialmente en trabajos didácticos (Todd et al. 2007; Farji-Brener 2012, 2013; Lortie 2013; Oesterheld 2013). Un artículo puede ser muy usado y tener fuerte influencia conceptual sobre el diseño de un estudio, pero ser poco citado. En muchos manuscritos (como hipotéticamente ocurre en este caso), el verdadero impacto es su capacidad de modificar las ideas o el desarrollo de las actividades académicas de sus lectores (Bornman et al. 2008). Segundo, estos resultados ilustran la importancia de publicar artículos en español sobre temáticas que colaboran al desarrollo de la investigación científica en países hispano-parlantes. Para que este tipo de artículo influya sobre el público hacia el que va dirigido, es muy valioso que estén escritos en el idioma en que la comunidad de estudiantes y científicos jóvenes tienen mejor capacidad de asimilar conceptos sutiles y complejos. Y esto es especialmente relevante a pesar de que esta clase de manuscritos sean subvalorados en las instancias formales de evaluación académica.

Agradecimientos. Los comentarios de 3 revisores anónimos y del editor ayudaron a mejorar una versión preliminar de este manuscrito.

\section{Referencias}

Bornmann, L., R. Mutz, C. Neuhaus, and H. Daniel. 2008. Citation counts for research evaluation: standards of good practice for analyzing bibliometrics data and presenting and interpreting results. Ethics Sci Environ Polit 8:93-102. https://doi.org/10.3354/esep00084.

Bunge, M. 2002. La investigación científica: su estrategia y su filosofía. Siglo XXI.

Farji-Brener, A. G. 2003. Uso correcto, parcial e incorrecto de los términos “hipótesis" y "predicciones" en ecología. Ecología Austral 13:223-227.

Farji-Brener, A. G. 2012. El valor de tener muchas citas. Ecología Austral 22:215-220. https://doi.org/10.25260/ EA.12.22.3.0.1229.

Farji-Brener, A. G. 2013. Tengo una cita: respuesta a Martín Oesterheld. Ecología Austral 23:74-76.

Farji-Brener, A. G. 2019. Una propuesta de marco conceptual para el desarrollo de proyectos de investigación en Entomología y ciencias afines. Revista Colombiana de Entomología 45(1):e7805-e7805. https://doi.org/10.25100/ socolen.v45i0.7805.

Farji-Brener, A. G., and S. Amador-Vargas. 2014. Hierarchy of hypotheses or cascade of predictions? A comment on Heger et al. Ambio 43(8):1112-1114. https://doi.org/10.1007/s13280-014-0549-0.

Farji-Brener, A. G., and S. Amador-Vargas. 2018. Hierarchy of hypotheses or hierarchy of predictions? Clarifying key concepts in ecological research. Invasion Biology: Hypotheses and Evidence 9:19. https://doi.org/10.1079/ 9781780647647.0019.

Feinsinger, P. 2013. Metodologías de investigación en ecología aplicada y básica: ¿Cuál estoy siguiendo y por qué? Revista Chilena de Historia Natural 86:385-402. https://doi.org/10.4067/S0716-078X2013000400002.

Feinsinger, P. 2014. El Ciclo de Indagación: una metodología para la investigación ecológica aplicada y básica en los 
sitios de estudios socio-ecológicos a largo plazo, y más allá. Bosque (Valdivia) 35:449-457. https://doi.org/10.4067/ S0717-92002014000300020.

Jaksic, F. M. 1994. Artículos clásicos, modas e impacto en ecología: los ecólogos chilenos en el contexto internacional, regional y local. Revista Chilena de Historia Natural 67:245-251.

Lawton, J. 1996. Patterns in ecology. Oikos 75:145-147. https://doi.org/10.2307/3546237.

Lortie, C. J., L. W. Aarssen, A. E. Budden, and R. Leimu. 2013. Do citations and impact factors relate to the real numbers in publications? A case study of citation rates, impact, and effect sizes in ecology and evolutionary biology. Scientometrics 94:675-682. https://doi.org/10.1007/s11192-012-0822-6.

Marone, L., and R. González Del Solar. 2000. Homenaje a Mario Bunge: o por qué las preguntas en Ecología deberían comenzar con por qué. Tópicos actuales en Filosofía de la ciencia: Homenaje a Mario Bunge en su 80 aniversario. Mar del Plata: Editorial Martín.

Marone, L., and L. Galetto. 2011. El doble papel de las hipótesis en la investigación ecológica y su relación con el método hipotético-deductivo. Ecología Austral 21:201-216.

Monroy, L., M. Rivera, and L. Dávila. 2018. Análisis estadístico de datos categóricos. Universidad Nacional de Colombia.

Oesterheld, M. 2013. El valor de tener muchas citas. Un comentario. Ecología Austral 23:70-73.

Olby, R. 2003. Quiet debut for the double helix. Nature 421:402-405. https://doi.org/10.1038/nature01397.

Todd, P. A., D. C. Yeo, D. Li, and R. J. Ladle. 2007. Citing practices in ecology: can we believe our own words? Oikos 116:1599-1601. https://doi.org/10.1111/j.0030-1299.2007.15992.x. 